Noname manuscript No.

(will be inserted by the editor)

\title{
Shigeki MATSUTANI \\ Gauss Optics and Gauss Sum on an Optical Phenomena
}

Received: date / Accepted: date

\begin{abstract}
In the previous article (Found Phys. Lett. 16 325-341), we showed that a reciprocity of the Gauss sums is connected with the wave and particle complementary. In this article, we revise the previous investigation by considering a relation between the Gauss optics and the Gauss sum based upon the recent studies of the Weil representation for a finite group.
\end{abstract}

Keywords Gauss reciprocity - wave-particle complementary $\cdot \operatorname{SL}(2, \mathbb{Z})$ • Weil representation

\section{Introduction}

In the previous article [25, we investigated a relation between the Gauss reciprocity and wave-particle complementary on an optical system, the fractional Talbot system [43] following the excellent work of Berry and Klein [3].

More precisely, in [25], we considered the wavy and particle-like treatments of the system, or treatments based upon the Helmholtz equation and the Fresnel integral. Both treatments express the same phenomena and the final results must agree; the agreement between them means a kind of the wave-particle complementary. As the fractional Talbot phenomena has discrete nature, the distribution at the screen expressed in terms of the Gauss sum parameterized by coprime integers $(p, q)[3$; the Gauss sum is a number theoretical function which is well-known in number theory (see \$2.1) and plays the central roles in both quadratic number theory and cyclotomic field theory 17. Corresponding to the agreement between the wavy and particle-like treatments, there appears a reciprocity between the Gauss sums

S. Matsutani

8-21-1 Higashi-Linkan, Sagamihara 228-0811

E-mail: rxb01142@nifty.com 
parameterized by both $(p, q)$ and $(q, p)$ [25], which is known as the Gauss reciprocity [15, Chap.8].

In 25] even though we dealt with the optical system, using the similarity between para-axial optics and non-relativistic quantum mechanics 13 , p.75-84], we have concluded that in the system, the canonical commutation relation in the quantum mechanics,

$$
q p-p q=i \hbar
$$

for position operator $q$ and momentum operator $p$, is similar to a relation in the primitive number theory that for coprime numbers $p$ and $q$ there exist integers $\left\{\frac{1}{q}\right\}_{p}$ and $\left\{-\frac{1}{p}\right\}_{q}$ such that

$$
p\left\{\frac{1}{p}\right\}_{q}-q\left\{-\frac{1}{q}\right\}_{p}=1 .
$$

The existence of these integers is primitively proved, e.g., 42, Theorem III.1] [17, p.4, Lemma 4] (see 82.1). Most of all theorems in number theory are based upon the relation (2) and it is regarded as a fundamental relation in number theory. It is very similar to the fact that (1) is the fundamental relation in the quantum mechanics. Both (11) and (2) play the central roles in the wave-particle complementary and the Gauss reciprocity respectively. In 25] a question arises why they appear and play the similar roles in the optical system. The purpose of this article is to answer this question.

On the other hand, in [41, Weil studied the symplectic group and the Gauss sum based upon development of the quantum mechanics, and found the Heisenberg group, its Schrödinger representation and a unitary representation of the metaplectic group known as the Weil representation. The Weil representation is essential to the foundation of quantum mechanics and linear optics. Following [41, Guillemin and Sternberg [13] and Raszillier and Schempp [30] gave physical interpretations of the Heisenberg group, the Schrödinger representation and the Weil representation in quantum mechanics and optics. Due to the discreteness and finiteness properties of the fractional Talbot system, the Heisenberg group related to the system becomes a finite group as we will show in 96 . Recently the relations between the Weil representation and the Gauss sum over a finite ring are studied well 4,7, 7, 34, 36, 39. In this article, we answer the question in [25] following these studies.

The fractional Talbot phenomena also has some influence on modern optics, quantum problems and materials physics, e.g., optical fibers [31, quantum information [2], the cyclotomic quantum clock problem [29], and composite metamaterials such as multilayer positive and negative optical index media 16. It is crucial to understand the algebraic essential of the fractional Talbot phenomena in terms of the Heisenberg group and the Weil representation. Then it enables us to answer a question how the discrete nature and the property of the finiteness influence the optical system.

Though it is historical irony, K. F. Gauss studied well the Gauss sum and wrote it in [1] 1818 and summarized the Gauss optics in [12 1840, in 
which he described the critical relation between $\operatorname{Sp}(2, \mathbb{R})=\mathrm{SL}(2, \mathbb{R})$ and optical system, whereas H. F. Talbot discovered the original Talbot phenomena around 1836 37, though the fractional Talbot phenomena was discovered by J. T. Winthrop and C. R. Worthington in 43. Talbot also studied elliptic integrals as a mathematician [6, p.413] and wrote several articles in the creation of the theory of elliptic functions [44. For example, he wrote about the "Abelian integral" in a letter of Sept. 8, 1844 to J. F. W. Herschel [38. It is well-known that Gauss also studied elliptic integrals and elliptic functions alone 6. The periodicity and algebraic structure in the elliptic integrals were the theme in the elliptic function theory. I will show that in the Talbot phenomena, the periodicity and algebraic structure also plays important role. In fact the Gauss sum and the optical system are formulated by the elliptic theta functions as we will show in (14). Further recent studies [10, 18, 39] show that the generalized theta functions and Gauss sum are connected in other physical problems e.g., Chern-Simons-Witten theory. Thus to consider relations among Gauss sum, Gauss optics, elliptic theta function and fractional Talbot phenomena is also interesting from the viewpoints of recent developments and science history.

Here we mention the contents of this article; Section 2 is devoted to the mathematical preliminary of the fundamental relation (2), the Gauss sum and the Gauss reciprocity. Section 3 is a review of the Gauss Optics and $\mathrm{SL}(2, \mathbb{R})$ based upon [13. In section 4 , following [20, we give the fractional Talbot phenomena in the framework of the Gauss optics and show the relation between the Gauss sum and Gauss optics. Section 5 is devoted to a review of the Heisenberg group and Weil representation following [13,30] and the recent movements $4,7,34,36,39$. In section 6 , as a revised investigation of the fractional Talbot phenomena in [25], we discuss these properties, especially algebraic properties of the the fractional Talbot phenomena. There we find a key fact to an answer of the question in [25. In section 7 , we will give an answer to the question using the key fact and further comments.

Here $\mathbb{R}, \mathbb{Q}$, and $\mathbb{Z}$ denote sets of real numbers, the fractional numbers, and integers respectively.

\section{Mathematical Preliminary}

This section is for the mathematical preliminary of the fundamental relation (2), the Gauss sum and the Gauss reciprocity.

\subsection{On the fundamental relation (2)}

In number theory, it is well known that there exist integers $\left\{\frac{1}{q}\right\}_{p}$ and $\left\{-\frac{1}{p}\right\}_{q}$ as in (44) such that

$$
p\left\{\frac{1}{p}\right\}_{q}-q\left\{-\frac{1}{q}\right\}_{p}=1
$$


This can be easily and elementally proved; for example see 42, Theorem III.1] and [17, p.4, Lemma 4]. This relation is a theoretical base of primitive number theory [17, 42 .

From (3), there uniquely exists a positive integer $\left[\frac{1}{p}\right]_{q}$ smaller than $q$ satisfying

$$
p\left[\frac{1}{p}\right]_{q} \equiv 1 \bmod q
$$

and we have

$$
p\left[\frac{1}{p}\right]_{q}+q\left[\frac{1}{q}\right]_{p}=1+p q .
$$

By letting

$$
\left[-\frac{1}{q}\right]_{p}:=p-\left[\frac{1}{q}\right]_{p},
$$

and for arbitrary $n \in \mathbb{Z},\left\{\frac{1}{q}\right\}_{p}$ and $\left\{-\frac{1}{p}\right\}_{q}$ in (3) are realized by

$$
\left\{\frac{1}{p}\right\}_{q}=\left[\frac{1}{p}\right]_{q}+n q, \quad\left\{-\frac{1}{q}\right\}_{p}=\left[-\frac{1}{q}\right]_{p}+n p .
$$

In other words, the number of pairs $\left(\left\{\frac{1}{p}\right\}_{q},\left\{\frac{1}{q}\right\}_{p}\right)$ satisfying (3) is countably infinite. As the $n=0$ case is essential, one can identify them with $\left[\frac{1}{p}\right]_{q}$ and $\left[-\frac{1}{q}\right]_{p}$ if needs.

\subsection{Quadratic theory}

The Legendre symbol $\left(\begin{array}{l}p \\ s\end{array}\right)$ for a prime number $s$ is defined by [17, Chap.5],

$$
\left(\begin{array}{l}
p \\
s
\end{array}\right):=\left\{\begin{array}{lc}
+1, & \text { if there is an integer } m \text { such that } m^{2}=p \bmod s, \\
-1, & \text { otherwise }
\end{array}\right.
$$

Further the Jacobi symbol for coprime numbers $p$ and $q$ is given by

$$
\left(\begin{array}{l}
p \\
q
\end{array}\right):=\prod_{s_{1}, \cdots, s_{n}}\left(\begin{array}{c}
p \\
s_{i}
\end{array}\right)
$$

where $s_{1}, \cdots, s_{n}$ are prime numbers such that $q=s_{1} \cdots s_{n}$.

Then the Jacobi quadratic reciprocity is well-known as [17, Prop. 5.2.2],

$$
\left(\begin{array}{l}
p \\
q
\end{array}\right)\left(\begin{array}{l}
q \\
p
\end{array}\right)=(-1)^{(p-1) / 2)(q-1) / 2}
$$




\subsection{Gauss sum}

Here I mention the Gauss sum primitively, though in the previous article 25, it is mentioned in detail. Though the Gauss sum can be defined using a multiple character and an additive character generally [17, Chap. 8 §2], we deal only with the quadratic Gauss sum, which is given by [17, Chap. 6],

$$
G(p, q, d):=\sum_{c=0}^{q-1} \mathrm{e}^{\frac{2 \pi i p}{q}(c+d)^{2}}
$$

where $q$ and $p$ are coprime integers and $d$ is an integer. (Using (3), we sometimes use another expression $G^{\prime}\left(p^{\prime}, q^{\prime}, d\right):=\sum_{c=0}^{q^{\prime}-1} \mathrm{e}^{\frac{\pi i p^{\prime}}{q^{\prime}}(c+d)^{2}}$ as mentioned in 25] but in this section, we employ the version with the $2 \pi$ prefactor in the exponent1. ) For simplicity, we consider $G(p, q):=G(p, q, 0)$ and the case that $q$ is an odd prime number. Since

$$
\sum_{c=0}^{q-1} \mathrm{e}^{\frac{2 \pi i p}{q} c}=0
$$

we have another representation using the Legendre symbol,

$$
G(p, q)=\sum_{c=0}^{q-1}\left(\begin{array}{c}
p c \\
q
\end{array}\right) \mathrm{e}^{\frac{2 \pi i p}{q} c}=\left(\begin{array}{c}
p \\
q
\end{array}\right) G(1, q), \quad G(1, q)=\sum_{c=0}^{q-1}\left(\begin{array}{l}
c \\
q
\end{array}\right) \mathrm{e}^{\frac{2 \pi i p}{q} c} .
$$

It is not difficult to prove that [17, Prop. 6.3.2],

$$
G(1, q)^{2}=(-1)^{(q-1) / 2} q .
$$

Thus it is a concerned problem to determine the sign in the Gauss sum [17, Chap. 6 §4] and then we obtain

$$
G(1, q)=\left\{\begin{array}{lll}
\sqrt{q}, & \text { if } q \equiv 1 \quad \text { module } 4 \\
i \sqrt{q}, & \text { if } q \equiv 3 & \text { module } 4 .
\end{array}\right.
$$

More general case $G(p, q, d)$ is mentioned well in [25, Appendix].

The Gauss reciprocity is studied well due to Hecke [15, Chap.8], which is explained in detail in [25],

$$
\frac{1}{|q|^{1 / 2}} \sum_{c \in \mathbb{Z} / q \mathbb{Z}} \mathrm{e}^{\frac{\pi i p}{q}(c+d)^{2}}=\mathrm{e}^{\frac{\pi i}{4} \operatorname{sgn}(p q)} \frac{1}{|p|^{1 / 2}} \sum_{c \in \mathbb{Z} / p \mathbb{Z}} \mathrm{e}^{-\frac{\pi i q}{p} c^{2}-2 \pi i d c} .
$$

Due to Weil representation [41, we could regard that the factor $\mathrm{e}^{\pi i / 4}$ is related to the Maslov index and phase anomaly [35, 5]

\footnotetext{
1 The correspondence between both expressions is simple for a case $p=2 p^{\prime}$. For other cases, we need subtle treatments. When $q^{\prime}$ is odd case, we find $\left[\frac{1}{2}\right]_{q^{\prime}} \in \mathbb{Z} / q^{\prime} \mathbb{Z}$ satisfying (4). More precise argument is left to [25].
} 


\section{Gauss Optics and $\operatorname{SL}(2, \mathbb{R})$}

Here let us review Gauss optics following the Guillemin and Sternberg 13 .

In 12, Gauss showed us that the optical system is recognized as a $\mathrm{SL}(2, \mathbb{R})=\operatorname{Sp}(2, \mathbb{R})$ map between incoming plane $S_{1}$ and outgoing screen $S_{2}$. For $c=1,2$, we choose the coordinate systems denoted by

$$
w_{c}:=\left(\begin{array}{l}
x_{c} \\
u_{c}
\end{array}\right) \in S_{c}
$$

where $u_{c}=d x_{c} / d z$ is the angle variable at $S_{c}$ respectively along the optical axis $z$. The origin of $x_{c}$ coincides with the optical axis. In the Gauss optics, i.e., two-dimensional linear optic:2, the optical system is represented by the special linear group

$$
\mathfrak{g} \in \mathrm{SL}(2, \mathbb{R}):=\left\{\left(\begin{array}{ll}
A & B \\
C & D
\end{array}\right) \mid A D-B C=1, A, B, C, D \in \mathbb{R}\right\}
$$

such that its action to $S_{c}$ is given by,

$$
w_{2}=\mathfrak{g} w_{1}
$$

In other words, every two-dimensional linear optical system (or Gaussian optical system) corresponds to an element $\mathrm{SL}(2, \mathbb{R})$ bijectively; $\left(\begin{array}{ll}1 & \delta z \\ 0 & 1\end{array}\right)$ corresponds to the translation by $\delta z$ along the optical axis whereas $\left(\begin{array}{ll}1 & 0 \\ P & 1\end{array}\right)$ to the thin lens with power $P$. The optical system consists of combination of various translations and thin lenses while $\mathrm{SL}(2, \mathbb{R})$ is generated by both matrices for appropriate $\delta z$ 's and $P$ 's. Every element $\mathfrak{g} \in \operatorname{SL}(2, \mathbb{R})$ preserves the symplectic product of

$$
\left\langle w_{1}, w_{1}^{\prime}\right\rangle=x_{1} u_{1}^{\prime}-x_{1}^{\prime} u_{1},
$$

i.e., $\left\langle w_{1}, w_{1}^{\prime}\right\rangle=\left\langle\mathfrak{g} w_{1}, \mathfrak{g} w_{1}^{\prime}\right\rangle$.

Now we fix an optical system and thus an element $\mathfrak{g} \in \mathrm{SL}(2, \mathbb{R})$ like (23). For the the system, we deal with the Lagrangian submanifold [13, p.34],

$$
S^{\mathrm{intf}}:=\left\{(w, \mathfrak{g} w) \mid w \in S_{1}\right\} \subset S_{1} \times S_{2} .
$$

Since $S^{\text {intf }}$ is known as two-dimensional manifold [13, in the interference phenomena, we pick up the independent variables $x_{1}$ and $x_{2}$ to express $S^{\text {intf }}$. In other words, $u_{c}(c=1,2)$ is a function of $x_{1}$ and $x_{2}$ as

$$
u_{1}=\frac{x_{2}-A x_{1}}{B}, \quad u_{2}=\frac{D x_{2}-x_{1}}{B} .
$$

\footnotetext{
${ }^{2}$ In [12, Gauss dealt with three dimensional optical system $(x, y, z)$ with cylindrical symmetry. $(x, d x / d z, y, d y / d z, z)$ was dealt with but the cylindrical symmetry reduces it to two dimensional linear optical system $(r, z)$ or $(r, d r / d z, z)$ for $r=\sqrt{x^{2}+y^{2}}$.
} 
Then the optical length is given by

$$
\begin{aligned}
L & =\frac{1}{2}\left\langle w_{1}, w_{2}\right\rangle+z_{2}-z_{1} \\
& =\frac{1}{2 B}\left(D x_{2}^{2}-2 x_{1} x_{2}+A x_{1}^{2}\right)+z_{2}-z_{1} .
\end{aligned}
$$

As in [13, we have the wave functions $\psi_{c}$ over $S_{c}(c=1,2)$ under the scalar approximation. For given $\psi_{1}$ over $S_{1}$, we have the image of $\psi_{2}$ at $S_{2}$;

$$
\begin{aligned}
\psi_{2}\left(x_{2}\right) & =\left(\frac{i}{\lambda B}\right)^{1 / 2} \exp \left(\frac{2 \pi i}{\lambda}\left(z_{1}-z_{2}\right)\right) . \\
& \int d x_{1} \psi_{1}\left(x_{1}\right) \exp \left(\frac{\pi}{B \lambda} i\left(D x_{2}^{2}-2 x_{1} x_{2}+A x_{1}^{2}\right)\right),
\end{aligned}
$$

where $\lambda$ is the wave length. We introduce $\phi_{2}$ by the relation,

$$
\psi_{2}\left(x_{2}\right)=\mathrm{e}^{\left(\frac{2 \pi i}{\lambda}\left(z_{1}-z_{2}\right)\right)} \phi_{2}\left(x_{2}\right)
$$

\section{Talbot phenomena}

In this section, we will review the fractional Talbot phenomena [3, 20, 25, 43 and consider a relation between the Gauss sum and the Gauss optics explicitly.

As in [3, 25], we will consider the $\delta$-comb grating plane $z=0$,

$$
\psi_{1}(x)=\sum_{n \in \mathbb{Z}} \delta(x-n a) .
$$

Here we note that there is a group action $\mathfrak{t}_{a}$ on $S_{c}$ :

$$
\mathfrak{t}_{a} \cdot\left(\begin{array}{l}
x \\
u
\end{array}\right)=\left(\begin{array}{c}
x+a \\
u-\frac{A}{2 B} a
\end{array}\right) .
$$

The $\delta$-comb gives the distribution at the screen,

$$
\phi_{2}\left(x_{2}\right)=\left(\frac{i}{\lambda B}\right)^{1 / 2} \sum_{n \in \mathbb{Z}} \exp \left(\frac{\pi}{B \lambda} i\left(D x_{2}^{2}-2 n a x_{2}+A n^{2} a^{2}\right)\right) .
$$

We write this by $\phi_{2}^{I}$.

Using the Poisson sum relation of (8),

$$
\psi_{1}\left(x_{1}, 0\right)=\sum_{n \in \mathbb{Z}} \frac{1}{a} \exp \left(2 \pi i \frac{x_{1} n}{a}\right)=\sum_{n \in \mathbb{Z}} \delta\left(x_{1}-a n\right)
$$

we have another expression of (10) 20]

$$
\phi_{2}\left(x_{2}\right)=\left(\frac{1}{A a^{2}}\right)^{1 / 2} \exp \left(\frac{\pi i x_{2}^{2}}{\lambda} C\right) \sum_{n \in \mathbb{Z}} \exp \left(\pi i\left(\frac{2 n x_{2}}{a A}-\frac{B \lambda n^{2}}{A a^{2}}\right)\right) .
$$


We write this by $\phi_{2}^{I I}$. In [25], we have obtained the essentially same as the expression (12) using the Helmholtz equation. As (12) comes from the $\frac{1}{a} \exp \left(2 \pi i \frac{x n}{a}\right)$ which exhibits wavy properties, we will regard (12) as the wavy expression. This is contrast to (10), which we are to consider as a particle-like expression.

Noting that $a^{2} / \lambda$ is the order of length and the unit of the system, we will scale the variables as,

$\hat{x}_{c}:=\frac{x_{c}}{a}, \quad \hat{u}:=u, \quad\left(\begin{array}{cc}\hat{A} & \hat{B} \\ \hat{C} & \hat{D}\end{array}\right):=\left(\begin{array}{cc}A & B a^{2} / \lambda \\ C \lambda / a^{2} & D\end{array}\right), \quad \hat{\phi}_{2}^{I, I I}\left(\hat{x}_{2}\right):=a \phi_{2}^{I, I I}\left(x_{2}\right)$.

Then we have the relations,

$$
\begin{aligned}
\hat{\phi}_{2}^{I}\left(\hat{x}_{2}\right) & =\left(\frac{i}{\hat{B}}\right)^{1 / 2} \sum_{n \in \mathbb{Z}} \exp \left(\frac{\pi}{\hat{B}} i\left(\hat{D} \hat{x}_{2}^{2}-2 n \hat{x}_{2}+\hat{A} n^{2}\right)\right) \\
\hat{\phi}_{2}^{I I}\left(x_{2}\right) & =\left(\frac{1}{\hat{A}}\right)^{1 / 2} \exp \left(\pi i \hat{x}_{2}^{2} \hat{C}\right) \sum_{n \in \mathbb{Z}} \exp \left(\pi i\left(\frac{2 n \hat{x}_{2}}{\hat{A}}-\frac{\hat{B} n^{2}}{\hat{A}}\right)\right) .
\end{aligned}
$$

As we mentioned in Introduction, they are written by the elliptic theta functions [32, p.35],[44, p.463]. By letting

$$
\tau:=\frac{\hat{B}}{\hat{A}},
$$

$\phi$ 's are written by

$$
\begin{gathered}
\hat{\phi}_{2}^{I}\left(\hat{x}_{2}\right)=\left(\frac{i}{\hat{B}}\right)^{1 / 2} \mathrm{e}^{\pi \frac{\hat{D}}{B} i \hat{x}_{2}^{2}} \theta\left(-\frac{\hat{x}_{2}}{\hat{B}} ; \tau\right), \\
\hat{\phi}_{2}^{I I}\left(\hat{x}_{2}\right)=\left(\frac{1}{\hat{A}}\right)^{1 / 2} \mathrm{e}^{\pi i \hat{C} \hat{x}_{2}^{2}} \theta\left(\tau \frac{\hat{x}_{2}}{\hat{B}} ;-\frac{1}{\tau}\right) .
\end{gathered}
$$

Here $\theta$ is the well-known theta function [32, p.35] [44, p.463],

$$
\theta(u, \tau):=\sum_{n \in \mathbb{Z}} \mathrm{e}^{\pi i\left(2 n u+\tau n^{2}\right)} .
$$

The system of the Talbot phenomena is written by the elliptic theta functior 3 . Then the equality,

$$
\hat{\phi}_{2}^{I}=\hat{\phi}_{2}^{I I}
$$

is interpreted as the Jacobi imaginary transformation in the elliptic theta functions [44, p.475].

Let us consider the fractional Talbot phenomena in the Gauss optics and its connection to the Gauss sums and the Gauss reciprocity. The ordinary Talbot phenomena was studied in [20].

\footnotetext{
3 As mentioned in Introduction, this historical meaning is very interesting.
} 
Let us consider the case

$$
\frac{A}{B} \frac{a^{2}}{\lambda}=\frac{\hat{A}}{\hat{B}}=\frac{p}{q}, \quad \kappa_{1}:=\frac{\hat{D}}{\hat{A}}, \quad \kappa_{2}:=\frac{1}{\hat{A}}, \quad \kappa_{3}:=\hat{C},
$$

where $p$ and $q$ are coprime numbers and

$$
\kappa_{3}=\frac{p}{q}\left(\frac{\kappa_{1}}{\kappa_{2}^{2}}-\kappa_{2}\right)
$$

Then we have

$$
\begin{aligned}
\hat{\phi}_{2}^{I}\left(\hat{x}_{2}\right) & =\left(\frac{i}{\hat{B}}\right)^{1 / 2} \sum_{n \in \mathbb{Z}} \exp \left(\frac{p}{q} \pi i\left(\kappa_{1}\left(\hat{x}_{2}\right)^{2}-2 \kappa_{2} n \hat{x}_{2}+n^{2}\right)\right) \\
\hat{\phi}_{2}^{I I}\left(\hat{x}_{2}\right) & =\left(\frac{1}{\hat{A}}\right)^{1 / 2} \exp \left(\pi i \kappa_{3}\left(\hat{x}_{2}\right)^{2}\right) \sum_{n \in \mathbb{Z}} \exp \left(\frac{1}{p} \pi i\left(2 n \kappa_{2} p \hat{x}_{2}-q n^{2}\right)\right) .
\end{aligned}
$$

For $n=r \ell+s$, we have

$$
\frac{1}{r}\left(K_{1} n+t n^{2}\right)=K_{1} \ell+2 t s \ell+t r \ell^{2}+\frac{1}{r}\left(K_{1} s+t s^{2}\right) .
$$

and thus

$$
\sum_{n \in \mathbb{Z}} \mathrm{e}^{\frac{\pi i}{r}\left(K_{1} n+t n^{2}\right)}=\sum_{\ell \in \mathbb{Z}} \sum_{s=0}^{r-1} \mathrm{e}^{\pi i\left(K_{1}+t r\right) \ell} \mathrm{e}^{\frac{\pi i}{r}\left(K_{1} s+t s^{2}\right)} .
$$

Here we used the fact $\mathrm{e}^{\pi i s \ell^{2}}=\mathrm{e}^{\pi i s \ell}$ and $\mathrm{e}^{2 t s \ell \pi i}=1$ for integers $s, t$ and $\ell$. Using these properties, $\phi_{2}$ 's become

$$
\begin{gathered}
\hat{\phi}_{2}^{I}\left(\hat{x}_{2}\right)=\left(\frac{i}{\hat{B}}\right)^{1 / 2} \mathrm{e}^{i \pi \frac{p}{q}\left(\kappa_{1} \hat{x}_{2}^{2}\right)} \sum_{\ell \in \mathbb{Z}} \mathrm{e}^{\pi i\left(2 \kappa_{2} p \hat{x}_{2}+p q\right) \ell} \sum_{s=0}^{p-1} \mathrm{e}^{\frac{\pi i}{q}\left(2 \kappa_{2} \hat{x}_{2} s+p s^{2}\right)}, \\
\hat{\phi}_{2}^{I I}\left(\hat{x}_{2}\right)=\left(\frac{1}{\hat{A}}\right)^{1 / 2} \mathrm{e}^{\pi i \kappa_{3} \hat{x}_{2}^{2}} \sum_{\ell \in \mathbb{Z}} \mathrm{e}^{\pi i\left(2 \kappa_{2} p \hat{x}_{2}+p q\right) \ell} \sum_{s=0}^{q-1} \mathrm{e}^{\frac{\pi i}{p}\left(2 \kappa_{2} p \hat{x}_{2} s+q s^{2}\right)} .
\end{gathered}
$$

As in [3, 25], the wave function of the system is rewritten as

$$
\hat{\phi}_{2}^{I, I I}\left(\hat{x}_{2}\right)=\sum_{n=-\infty}^{\infty} \mathcal{A}^{I, I I}(n ; q, p) \delta\left(\kappa_{2} \hat{x}_{2}-\frac{1}{2} e_{q p}-\frac{n}{q}\right),
$$

where

$$
e_{q p}:=\left\{\begin{array}{l}
1, \text { if } q p \text { odd } \\
0, \text { if } q p \text { even }
\end{array}\right.
$$


By choosing an appropriate prefactor, we have

$$
\begin{aligned}
\mathcal{A}^{I}(n ; q, p) & =\sqrt{\frac{i}{p}} \sum_{s=0}^{p-1} \exp \left(i \pi\left[\left(2 n+q e_{q p}\right) s+q s^{2}\right] / p+\hat{\kappa}_{1}\left(2 n+q e_{q p}\right)^{2} / 4 p q\right), \\
\mathcal{A}^{I I}(n ; q, p) & =\sqrt{\frac{1}{q}} \sum_{s=0}^{q-1} \exp \left(i \pi\left[\left(2 n+q e_{q p}\right) s-p s^{2}\right] / q+\hat{\kappa}_{3}\left(2 n+q e_{q p}\right)^{2} / 4 q^{2}\right),
\end{aligned}
$$

where

$$
\hat{\kappa}_{1}:=\frac{\kappa_{1}}{\kappa_{2}^{2}}=\hat{A} \hat{D}, \quad \hat{\kappa}_{3}:=\frac{\kappa_{3}}{\kappa_{2}^{2}}=\hat{A}^{2} \hat{C}
$$

Provided that $\hat{\kappa}_{1}$ and $\hat{\kappa}_{3}$ are some integers (or, more precisely speaking, certain fractional numbers), these are merely the Gauss sums. It implies that there appears the fractional Talbot phenomena in the Gauss optics, even though [20] argued only the integral case or $q / p=1$ case.

We should note that the equality between $\hat{\phi}_{2}^{I}$ and $\hat{\phi}_{2}^{I I}$ in (13) means the reciprocity,

$$
\hat{\phi}_{2}^{I}=\hat{\phi}_{2}^{I I}, \quad \mathcal{A}_{2}^{I}=\mathcal{A}_{2}^{I I} .
$$

As shown in 25], it means the Gauss reciprocity (6) [15. In other words, in the case, the Gauss optics, the Gauss sums, and the Gauss reciprocity are connected in the fractional Talbot system.

For the ordinary fractional Talbot phenomena case,

$$
\left(\begin{array}{ll}
\hat{A} & \hat{B} \\
\hat{C} & \hat{D}
\end{array}\right)=\left(\begin{array}{cc}
1 & q / p \\
0 & 1
\end{array}\right)
$$

$\mathcal{A}^{I}(n ; q, p)$ is given by [25],

$$
\left\{\begin{array}{c}
\left(\begin{array}{l}
p \\
q
\end{array}\right) \exp \left(i \pi\left[\frac{1}{4}(q-1)-\left(\frac{q}{p}\left(\left[\frac{1}{q}\right]_{p}\right)^{2}-\frac{1}{q p}\right) n^{2}\right]\right) \\
\left(\begin{array}{l}
q \\
p
\end{array}\right) \exp \left(-i \pi\left[\frac{1}{4} p+\left(\frac{q}{p}\left(\left[\frac{1}{q}\right]_{p}\right)^{2}-\frac{1}{q p}\right) n^{2}\right]\right) \\
\left(\begin{array}{l}
q \\
p
\end{array}\right) \exp \left(-i \pi\left[\frac{1}{4} p\right.\right. \\
\left.\left.+\left(\frac{2 q}{p}\left[\frac{1}{2}\right]_{p}\left[\frac{1}{2 q}\right]_{p}-\frac{1}{4 q p}\right)(2 n+q)^{2}\right]\right)
\end{array}\right.
$$


whereas $\mathcal{A}^{I I}(n ; q, p)$ is given by

$$
\left\{\begin{array}{c}
\left(\begin{array}{l}
p \\
q
\end{array}\right) \exp \left(i \pi\left[\frac{1}{4}(q-1)+\frac{p}{q}\left(\left[\frac{1}{p}\right]_{q}\right)^{2} n^{2}\right]\right) \\
\left(\begin{array}{l}
q \\
p
\end{array}\right) \exp \left(-i \pi\left[\frac{1}{4} p-\frac{p}{q}\left(\left[\frac{1}{p}\right]_{q}\right)^{2} n^{2}\right]\right) \\
\left(\begin{array}{l}
p \\
q
\end{array}\right) \exp \left(i \pi\left[\frac{1}{4}(q-1)+\frac{2 p}{q}\left[\frac{1}{2}\right]_{q}\left(\left[\frac{1}{2 p}\right]_{q}\right)^{2}(2 n+q)^{2}\right]\right)
\end{array}\right.
$$

where both are for " $p$ even, $q$ odd", " $p$ odd, $q$ even", and " $p$ odd, $q$ odd" respectively.

\section{Heisenberg Group and Schrödinger representation}

Here we review the Weil representation in order to answer the question why the Gauss sum appears in the optical system.

Let us consider a ring $R$ and $S:={ }^{t}(R, R)$. We assume that $R$ is $\mathbb{R}$, $\mathbb{Z}, \mathbb{Q}$ or $\mathbb{Z} / b \mathbb{Z}$, where $b$ is a positive odd number. The case that $R=\mathbb{R}$ is studied well in [13,30 for the optical system based upon [21,41] and thus so in this article, we basically assume that $R$ is $\mathbb{Z} / b \mathbb{Z}$. When $R$ is the finite ring, the Weil representation and Heisenberg group are recently studied well 4,7, 34, 36. In this article, we will consider only the simplest case and so if one considers more complicate cases, [4,7,34,36] are nice for the purpose and may provide the guide.

\subsection{Heisenberg Group}

Let us consider the Heisenberg group $H$ associated with $S=R^{2}$ and $Z=R$ [4,7, 34, 36,

$$
H:=(S, Z)
$$

with the product $H \times H \rightarrow H$ defined by

$$
\left(\hat{w}_{1}, z_{1}\right)\left(\hat{w}_{2}, z_{2}\right)=\left(\hat{w}_{1}+\hat{w}_{2}, z_{1}+z_{2}+\frac{1}{2}\left\langle\hat{w}_{1}, \hat{w}_{2}\right\rangle\right),
$$

for $\left(\left(\hat{w}_{1}, z_{1}\right),\left(\hat{w}_{2}, z_{2}\right)\right) \in H \times H$. It is obvious that the product is well-defined and it becomes a group.

The Heisenberg group is also characterized by an central extension of the Abelian group (free $R$-module) as [32, p.17],

$$
0 \rightarrow Z \rightarrow H \rightarrow R^{2} \rightarrow 0
$$

such that $e: R^{2} \times R^{2} \rightarrow Z$ by symplectic product $\frac{1}{2}\left\langle\hat{w}_{1}, \hat{w}_{2}\right\rangle$ for $\left(\left(\hat{w}_{1}, z_{1}\right),\left(\hat{w}_{2}, z_{2}\right)\right) \in$ $H \times H$.

Then we have the following facts: 
1. For $h_{1}, h_{2} \in H, h_{2} h_{1} h_{2}^{-1}=\left(\hat{w}_{1}, z_{1}+\left\langle\hat{w}_{1}, \hat{w}_{2}\right\rangle\right)$.

2. $N:=\{((0, u), z) \mid x, z \in R\}$ is a normal Abelian subgroup of $H$.

3. $Z:=\{((0,0), z) \mid z \in R\}, U:=\{((0, u), 0) \mid u \in R\}$, and $X:=$ $\{((x, 0), 0) \mid x \in R\}$ are normal Abelian subgroups of $H$ respectively.

4. $H=N \rtimes X$.

For $\gamma \in R^{\times}$, we have the action $R^{\times}$on $H$,

$$
\alpha_{\gamma} \cdot(w, z)=\left(\gamma w, \gamma^{2} z\right),
$$

which is regarded as an element of $\operatorname{Aut}(H)$. On the other hand, $\mathfrak{g} \in \mathrm{SL}(2, R)$ induces the automorphism of $H$,

$$
\mathfrak{g} \cdot(w, z)=(\mathfrak{g} w, z),
$$

or $\mathrm{SL}(2, R) \subset \operatorname{Aut}(H)$. When $R=\mathbb{R}$, we have $\mathrm{SL}(2, R) \cap R^{\times}=\{ \pm 1\}$. For $R=\mathbb{R}$ case, we have an exact sequence of topological groups,

$$
1 \rightarrow R^{\times} \rightarrow \mathrm{Mp}(2, R) \rightarrow \mathrm{Sp}(2, R) \rightarrow 1,
$$

where $\operatorname{Mp}(2, R)$ is the metaplectic group.

When $R$ is a finite ring $\mathbb{Z} / b \mathbb{Z}$, were $b$ are a positive integer, the Heisenberg group becomes a finite group. We will consider the automorphism in the group ring $\mathbb{C}[H]$.

\subsection{Character of Heisenberg Group}

When we regard $\mathbb{C}[H]$ as $\mathbb{C}[N]$-module, we apply the Mackey theory of the finite group [8, 2, 33] to it. We recall the Mackey theory which is given as follows:

Proposition 1 Let $K$ is an arbitrary field and $G$ be a finite group. Let $M$ be a simple $K[G]$ - module and $H$ be a normal subgroup of $G$. As $M$ can be regarded as $K[H]$-module, we denote it by $M_{H}$. Then followings hold

1. $M_{H}$ is completely reducible.

2. The irreducible $K[H]$-submodules of $M_{H}$ are all conjugates of each other.

$$
M_{H} \approx L^{\left(g_{1}\right)} \oplus L^{\left(g_{2}\right)} \oplus \cdots \oplus L^{\left(g_{r}\right)} .
$$

3. There are a subgroup $S$ of $G$, called inertia group, and $K[H]$-module $L$ such that for $g_{i} \in S, g_{i} L=L^{\left(g_{i}\right)}$ and $|S|=r$.

We have its character $\varrho_{\eta}: Z \rightarrow \mathbb{C}^{\times}$parameterized by $\eta \in \mathbb{R}, \quad$ e.g., $\eta=b$,

$$
\varrho_{\eta}(z)=\exp \left(\frac{2 \pi}{\eta} i z\right)
$$

As $N$ is Abelian, the natural projection $\varpi: N \rightarrow Z$ is a group homomorphism and thus we define $\varrho_{\eta}: N \rightarrow \mathbb{C}^{\times}$by,

$$
\varrho_{\eta}(n):=\varrho_{\eta} \circ \varpi(n) .
$$


For $n \in N$ and $h^{\prime} \in H$, we have a natural action on $\varrho_{\eta} \in \operatorname{Hom}\left(Z, \mathbb{C}^{\times}\right)$,

$$
\left(h \circ \varrho_{\eta}\right)(n):=\varrho_{\eta}\left(h^{\prime} \cdot n \cdot h^{\prime-1}\right)=\exp \left(\frac{2 \pi}{\eta} i\left(z+\left\langle w^{\prime}, w\right\rangle\right)\right) .
$$

Noting $\langle X, U\rangle \not \equiv 0$, and $\langle X, X\rangle=\langle U, U\rangle=0$, we may regard that $X$ has an action on $N^{\wedge}:=\operatorname{Hom}\left(N, \mathbb{C}^{\times}\right)$.

For $\varrho_{\eta} \in N^{\wedge} / X$, we consider $X_{\varrho}(\subset X)$ as the stablizar to $\varrho_{\eta}$. When $\varrho_{\eta}$ is trivial, $X_{\varrho}$ is equal to $X$ and then, the representation becomes $R^{2}$.

On the other hand, if $\varrho_{\eta}$ is a non-trivial case, $X_{\varrho}$ is equal to $\{0\}$ and then we consider the induced representation $\operatorname{ind}_{N}^{H}\left(\varrho_{\eta}\right)$.

We should note that $N$ is a normal subgroup $N \triangleleft H$ and thus we apply the Proposition to this system,

$$
\mathbb{C}[H] \approx \mathbb{C}\left[N_{1}\right] \oplus \mathbb{C}\left[N_{2}\right] \oplus \cdots \oplus \mathbb{C}\left[N_{b^{2}-1}\right] \oplus \mathbb{C}\left[N_{b^{2}}\right]=\oplus_{x \in X} \mathbb{C}[x N] .
$$

Here $\mathbb{C}\left[N_{i}\right]$ is $\mathbb{C}[N]$-module and $X$ is the inertia group. For $h=x n$ of $x \in X$, $n \in N$, we have

$$
\varrho_{i}(h)=\operatorname{tr} \mathcal{S}(h):=\left\{\begin{array}{cc}
\varrho_{\rho}(z) & \text { for } h=(0,0, z) \\
0 & \text { otherwise }
\end{array} .\right.
$$

We will consider a function over $H$, or an element of $(\chi(0), \chi(1), \cdots, \chi(b-1))$ belonging to $\oplus_{x \in X} \mathbb{C}[x N] \approx \mathbb{C}[H]$. By checking the action of $X, U$ and $Z$, we have the Schrödinger representation of $H$ which is generated by

or

$$
\begin{gathered}
\mathcal{S}(x) \chi\left(\left(x^{\prime}, u^{\prime}, z^{\prime}\right)\right)=\chi\left(\left(x+x^{\prime}, u^{\prime}, z^{\prime}\right)\right), \\
\mathcal{S}(u) \chi\left(\left(x^{\prime}, u^{\prime}, z^{\prime}\right)\right)=\mathrm{e}^{\frac{2 \pi i}{\eta} u x^{\prime}} \chi\left(\left(x^{\prime}, u^{\prime}, z^{\prime}\right)\right), \\
\mathcal{S}(z) \chi\left(\left(x^{\prime}, u^{\prime}, z^{\prime}\right)\right)=\mathrm{e}^{\frac{2 \pi i}{\eta} z} \chi\left(\left(x^{\prime}, u^{\prime}, z^{\prime}\right)\right),
\end{gathered}
$$

$$
\mathcal{S}(x)=\left(\begin{array}{ccccc}
0 & 1 & & & \\
& 0 & 1 & & \\
& & 0 & \ddots & \\
& & \ddots & 1 \\
& & & 0 & 1 \\
& & & & 0
\end{array}\right)^{x}, \quad \mathcal{S}(u)=\left(\begin{array}{llll}
1 & & & \\
& \mathrm{e}^{2 \pi i u / \eta} & & \\
& & \ddots & \\
& & & \mathrm{e}^{2 \pi(b-1) i u / \eta}
\end{array}\right) .
$$

When $R=\mathbb{R}$, we consider $L^{2}(\mathbb{R})$ instead of $\mathbb{C}[X]$ and then we could define $d W(\xi), \xi \in \mathfrak{h}$ for the Lie algebra $\mathfrak{h}$ of $H$,

$$
d \mathcal{S}(\xi) \chi=\left.\frac{d}{d t} \mathcal{S}(t \xi) \chi\right|_{t=0}
$$

Due to [21, 15], we have

$$
d \mathcal{S}\left(\xi_{x}\right)=\frac{d}{d x}, \quad d \mathcal{S}\left(\xi_{u}\right)=\frac{2 i \pi}{\eta} x, \quad d \mathcal{S}\left(\xi_{z}\right)=\frac{2 i \pi}{\eta} i d .
$$

This means that $\mathcal{S}(x)=\mathrm{e}^{x d \mathcal{S}\left(\xi_{x}\right)}, \mathcal{S}(u)=\mathrm{e}^{u d \mathcal{S}\left(\xi_{u}\right)}$, and $\mathcal{S}(z)=\mathrm{e}^{z d \mathcal{S}\left(\xi_{z}\right)}$. We have the canonical commutation relation,

$$
\left[d \mathcal{S}\left(\xi_{x}\right), d \mathcal{S}\left(\xi_{u}\right)\right]=d \mathcal{S}\left(\xi_{z}\right), \quad \text { or } \quad \frac{d}{d x} x-x \frac{d}{d x}=1 .
$$




\subsection{Representation of Automorphism of Heisenberg Group}

We mention the representation of $\operatorname{Aut}(H)$ for the Schrödinger representation $\mathcal{S}$. First we deal with the action $\alpha_{\gamma}$ of $R^{\times}$,

$$
\alpha_{\gamma} \cdot \mathcal{S}(h)=\mathcal{S}\left(\alpha_{\gamma}(h)\right)=\gamma^{2} \mathcal{S}(h) .
$$

Secondary we consider the $\mathfrak{g} \in \mathrm{SL}(2, R)$. By letting

$$
\mathfrak{g} \circ \mathcal{S}(h)=\mathcal{S}\left(\mathfrak{g}^{-1} h\right),
$$

it is shown that there exists the unitary action $W(\mathfrak{g})$ on $H$ such that

$$
\mathcal{S}(\mathfrak{g} h)=\mathcal{W}(\mathfrak{g}) \mathcal{S}(h) \mathcal{W}(\mathfrak{g})^{-1},
$$

for every $h \in H$ when $\mathbb{C}[H]$ is regarded as $\mathbb{C}[H]$-module. By tuning the factor, we obtain the Weil-representation of the metaplectic group $\mathrm{Mp}(2, R)$.

Following the case $R=\mathbb{R}\left[30\right.$, (3.15)], the Weil representation $[\mathcal{W}(\mathfrak{g}) \chi]\left(\hat{x}_{2}\right)$ is given by

$$
\begin{aligned}
& \sum_{\hat{x}_{1} \in R} G\left(\hat{x}_{2} ; \hat{x}_{1}\right) \chi\left(\hat{x}_{1}\right) \\
& =\sqrt{\frac{A i}{B \eta}} \sum_{\hat{x}_{1} \in R,\left(\hat{x}_{2}, \hat{u}_{2}\right)=\mathfrak{g}\left(\hat{x}_{1}, \hat{u}_{1}\right)} \exp \left(\frac{2 \pi}{\eta} i\left(\frac{1}{2}\left\langle\left(\hat{x}_{1}, \hat{u}_{1}\right),\left(\hat{x}_{2}, \hat{u}_{2}\right)\right\rangle\right)\right) \chi\left(\hat{x}_{1}\right),
\end{aligned}
$$

where $\hat{u}_{c}=\hat{u}_{c}\left(\hat{x}_{1}, \hat{x}_{2}\right)(c=1,2)$. Here $G\left(\hat{x}_{2} ; \hat{x}_{1}\right)$ has its multiplication

$$
G\left(\hat{x}_{3} ; \hat{x}_{1}\right)=\sum_{\hat{x}_{2} \in R} G\left(\hat{x}_{3} ; \hat{x}_{2}\right) G\left(\hat{x}_{2} ; \hat{x}_{1}\right) .
$$

The phase of $\frac{\hat{A} i}{\hat{B} \eta}(29)$ is given by

$$
s(\mathfrak{g})=\operatorname{sgn}(\hat{B}) \mathrm{e}^{\pi i / 2} .
$$

Hence $\mathcal{W}$ is the representation of the metaplectic group $\operatorname{Mp}(2, R)$.

We note that for example as in the path integral [35], the computation of the kernel function $G\left(\hat{x}_{2} ; \hat{x}_{1}\right)$ is based upon the canonical commutation relation (28).

\section{Gauss sum in fractional Talbot phenomena, revised}

In this section, we will investigate the relation between the Gauss sum and the fractional Talbot phenomena again more algebraically. This is a revised investigation of 25]. In other words, we consider why the optical system is expressed by the Gauss sums. We have to consider the symmetries of the system which insert the discrete pictures in the optical system and give an answer the question why (1) is similar to (2).

Let us reconsider the physical situations in $\S 2$ and $\S 3$. 


\subsection{The translation action $\mathfrak{t}_{a}^{\mathfrak{g}}$}

Here we will consider the first discrete nature in the Talbot phenomena coming from the delta-comb slit; due to it, the system is represented by theta function. Let us fix $\mathfrak{g} \in \mathrm{SL}(2, \mathbb{R})$, which means that we choose an optical system.

As the Lagrange submanifold $S^{\text {intf }}$ is now two-dimension, the parameters $\hat{x}_{1}$ and $\hat{x}_{2}$ of $\hat{w}_{2}=\mathfrak{g} \hat{w}_{1}$ are its local coordinates of $S^{\text {intf }}$ and thus $\hat{u}_{c}$ is expressed by [13, p.34],

$$
\hat{u}_{c}=\hat{u}_{c}\left(\hat{x}_{1}, \hat{x}_{2}, \mathfrak{g}\right), \quad \text { for } c=1,2 .
$$

We are concerned with the interference system $S^{\text {intf }}$ with the translation symmetry (9). The action of translation (9) induces

$$
\left(\begin{array}{l}
\hat{x}_{1} \\
\hat{u}_{1}
\end{array}\right)=\left(\mathfrak{t}_{a}^{\mathfrak{g}}\right)^{n}\left(\begin{array}{c}
\hat{x}_{0} \\
\hat{u}_{0}
\end{array}\right)=\left(\begin{array}{c}
\hat{x}_{0} \\
\hat{u}_{0}
\end{array}\right)+\left(\begin{array}{c}
n \\
-\frac{\hat{A}}{\hat{B}} n
\end{array}\right),
$$

so that it preserves $\hat{x}_{2}$ as $x$ component of image of $\mathfrak{g}$, i.e.,

$$
\left(\begin{array}{c}
\hat{x}_{2} \\
\hat{u}_{2}
\end{array}\right)=\mathfrak{g} \cdot\left(\mathfrak{t}_{a}^{\mathfrak{g}}\right)^{n}\left(\begin{array}{c}
\hat{x}_{0} \\
\hat{u}_{0}
\end{array}\right)=\mathfrak{g}\left(\begin{array}{c}
\hat{x}_{0} \\
\hat{u}_{0}
\end{array}\right)-\left(\begin{array}{c}
0 \\
\frac{1}{\hat{B}} n
\end{array}\right),
$$

which provides

$$
\frac{1}{2}\left\langle\mathfrak{t}_{a}^{\mathfrak{g} n} \hat{w}_{1}, \mathfrak{g t}_{a}^{\mathfrak{g} n} \hat{w}_{1}\right\rangle=\frac{1}{2 \hat{B}}\left(D \hat{x}_{2}^{2}-2\left(\hat{x}_{0}+n\right) \hat{x}_{2}+\hat{A}\left(\hat{x}_{0}+n\right)^{2}\right) .
$$

The above translation means that we deal with

$$
H_{\hat{w}_{0}}^{(a, \mathfrak{g})}:=\left\{(\hat{w}, z) \mid w=\mathfrak{t}_{a}^{\mathfrak{g} n} \hat{w}_{0}, n=0,1, \cdots, b-1\right\} .
$$

over $R=\mathbb{Q}\left[\left[\hat{A}, \hat{D}, 1 / \hat{B}, x_{0}, u_{0}\right]\right]$, a formal power series of $\hat{A}, \hat{D}, 1 / \hat{B}, x_{0}$ and $u_{0}$ over $\mathbb{Q}$. Here we should note that for $\hat{w}=(0,0)$ case, $H_{0}^{(a, \mathfrak{g})}:=\{(n,-\hat{A} / \hat{B} n, z)$ | $n \in \mathbb{Z}, z \in R\}$ is a normal subgroup of $H$ over $R=\mathbb{Q}\left[\left[\hat{A}, \hat{D}, 1 / \hat{B}, x_{0}, u_{0}\right]\right]$. $\mathbb{C}\left[H_{\hat{w}_{0}}^{(a, \mathfrak{g})}\right]$ is $\mathbb{C}\left[H_{0}^{(a, \mathfrak{g})}\right]$-module. Hence this discrete system consists of $H$ itself. The translation does not break the algebraic structure of the optical system given by [13,30].

From (14), we have the theta function expression due to the symplectic structure. In fact, $\left(\begin{array}{c}n \\ -\frac{\hat{A}}{B} n\end{array}\right)$ is written by $\left(\begin{array}{c}n \\ -\tau n\end{array}\right)$ which shows the periodic structure in the Abelian variety of genus one [32] $]^{4}$. Oskolkov [28] and Berry and Bodenschatz [1] dealt with different $\tau$ as time development and showed interesting patterns.

\footnotetext{
${ }^{4}$ It is very interesting that Talbot himself studied the Abelian integral [6, p.413].
} 
6.2 Discrete nature in the optical system

Here we will consider the second discrete nature in the Talbot phenomena. In order to insert another discrete nature $\mathbb{Q}$ in this system, we have imposed the condition (15),

$$
\frac{\hat{A}}{\hat{B}}=\frac{p}{q},
$$

where $p$ and $q$ are coprime numbers. Let us consider realization of (15) or (30) in $\operatorname{SL}(2, \mathbb{Z})$ and then we naturally encounter the simplest case,

$$
\left(\begin{array}{cc}
p & q \\
\left\{-\frac{1}{q}\right\}_{p} & \left\{\frac{1}{p}\right\}_{q}
\end{array}\right) \in \mathrm{SL}(2, \mathbb{Z})
$$

where

$$
\operatorname{det}\left(\begin{array}{cc}
p & q \\
\left\{-\frac{1}{q}\right\}_{p} & \left\{\frac{1}{p}\right\}_{q}
\end{array}\right)=p\left\{\frac{1}{p}\right\}_{q}-q\left\{-\frac{1}{q}\right\}_{p}=1 .
$$

This recovers (21) and then (21) becomes

$$
\kappa_{1}=p\left\{\frac{1}{p}\right\}_{q}, \quad \kappa_{3}=p^{2}\left\{-\frac{1}{q}\right\}_{p},
$$

and then $\mathcal{A}_{I}$ is represented by the Gauss sum explicitly.

The above condition corresponds to the ordinary fractional Talbot phenomena case (23) in $\operatorname{SL}(2, \mathbb{Q}) \subset \mathrm{SL}(2, \mathbb{R})$,

$$
\left(\begin{array}{cc}
1 & \hat{q} / p \\
0 & 1
\end{array}\right)=\left(\begin{array}{cc}
\frac{1}{p} & 0 \\
\left\{\frac{1}{q}\right\}_{p} & p
\end{array}\right)\left(\begin{array}{cc}
p & q \\
\left\{-\frac{1}{q}\right\}_{p} & \left\{\frac{1}{p}\right\}_{q}
\end{array}\right) \in \operatorname{SL}(2, \mathbb{Q}) .
$$

Using this fact, we will give an answer to the question in Introduction and [25] in next section.

\subsection{The fractional Talbot phenomena and Weil representation}

By inserting the discrete nature with the translation properties and $\mathrm{SL}(2, \mathbb{Z})$ into the Gauss optics in the previous subsections, we encounter $\mathrm{e}^{2 \pi i / q}$.

Here we will give its connection with the Weil representation in the previous section in order to consider the Gauss sum again.

Suppose that $R=\mathbb{Z} / q \mathbb{Z}$, and $\hat{x}_{0}, \hat{u}_{0}$ are elements of $R$. For simplicity, $q$ is an odd number. The character $\chi_{q}$ of $R$ is given by $\mathrm{e}^{2 \pi i / q}$. In order to choose $\hat{B} \in R^{\times}$freely, we restrict the group $\mathfrak{g}$ belonging to

$$
\Gamma(2, R):=\left\{\mathfrak{g}:=\left(\begin{array}{cc}
\hat{A} & \hat{B} \\
0 & \hat{C}
\end{array}\right) \mid \mathfrak{g} \in \mathrm{SL}(2, R)\right\},
$$


and we set $\hat{A} / \eta \hat{B}=q / p$. More specially, when we set

$$
\mathfrak{g}=\left(\begin{array}{ll}
1 & 1 \\
0 & 1
\end{array}\right), \quad \eta=\frac{q}{p}, \quad \chi\left(\hat{x}_{1}\right) \equiv 1 \text { for every } \hat{x}_{1},
$$

$[\mathcal{W}(\mathfrak{g}) \chi]\left(\hat{x}_{2}\right)$ in (29) is equal to

$$
\sqrt{\frac{q i}{p}} \sum_{\hat{x}_{1} \in R} \exp \left(\frac{q \pi}{p} i\left(\hat{x}_{2}^{2}-2\left(\hat{x}_{0}+n\right) \hat{x}_{2}+\left(\hat{x}_{0}+n\right)^{2}\right)\right) .
$$

By letting $\hat{x}_{2}=\left(q e_{p q}-2 n\right) / 2 q$, this is essentially the same as $\mathcal{A}_{2}^{I}$ in (32) of $\mathfrak{g}=\left(\begin{array}{cc}1 & q / p \\ 0 & 1\end{array}\right)$.

Then we realize $\mathcal{A}_{2}^{I}$ as in (32). Using the reciprocity for Gauss sums (6) or the reciprocity corresponding to the wave-particle complementarity (22), we also realize $\mathcal{A}_{2}^{I I}$ (25).

\section{Discussion}

In this article, we dealt with the Gauss optics with the delta-comb and we gave explicit expressions in terms of the theta functions (14). After considering the fractional condition,

$$
\frac{\hat{A}}{\hat{B}}=\frac{p}{q} \in \mathbb{Q},
$$

we expressed the fractional Talbot phenomena in the Gauss optics on $\mathfrak{g} \in$ $\mathrm{SL}(2, \mathbb{Q})$ explicitly as in $(20)$, and gave their relations to the Gauss sums and the Gauss reciprocity. Due to the $\mathrm{SL}(2, R)$ treatment which corresponds to the Gauss optics, we could argue the Weil representation and the Heisenberg group in the optical system [13,30].

When $R$ is continuous case or $\mathbb{R}$, the Heisenberg group is a Lie group and we have its Lie algebra. In the Schrödinger representation, the Lie algebra is generated by $x, \frac{d}{d x}$ and 1 with the canonical commutation relation as the generating relation of the algebra (28) [21,

$$
\frac{d}{d x} x-x \frac{d}{d x}=1 .
$$

The relation governs the kernel functions and automorphism of the Heisenberg group like $G\left(\hat{x}_{2} ; \hat{x}_{1}\right)$ in (29). The automorphism corresponds to the dynamics and time development in the quantum mechanics; in the case of the optics, it corresponds to the translation along the optical axis. Thus (33) is the fundamental relation in the automorphism.

On the other hand, when $R$ is $\mathbb{Z} / q \mathbb{Z}$ case, the Heisenberg group is a finite group and thus we can not deal with its infinitesimal difference neither its Lie algebra. Thus we must directly consider the automorphism of the 
Heisenberg group. Instead of the canonical commutation relation (28), we have the relation (31),

$$
p\left\{\frac{1}{p}\right\}_{q}-q\left\{-\frac{1}{q}\right\}_{p}=1,
$$

as the fundamental relation of the automorphism of the Heisenberg group, $\operatorname{SL}(2, \mathbb{Z}) \subset \operatorname{Aut}(H)$. As the effect of wavelength $\lambda$ is normalized in the relation (15), in (34) the wavy properties as the interference condition are built in. Thus (34) implicitly connects the linear optical property, i.e., of an element of $\operatorname{SL}(2, R)$, and the wavy property, i.e., $R=\mathbb{Z}$.

Hence we conclude that behind the fractional Talbot phenomena, these relations (33) and (34) exist and both play the same role essentially for the continuous case and for the discrete case. This means that we find the answer to the question in 25 .

We expect that this algebraic treatment of the Talbot phenomena has some effects on several fields related to quantum mechanics, the optical system, and missing relations between quantum mechanics and arithmetic theory [27, p.149][40].

As in the adelic consideration [40, Introduction], the $p$-adic quantum mechanics and ordinary quantum mechanics are treated equivalently. Then our interpretation of the relation between (33) and (34) is consistent with the philosophy of the adelic consideration because in $p$-adic quantum mechanics, $p$ is the small parameter associated with the $p$-adic differential operator [40].

Further as in survey of Polishchuk [32, the Gauss sum and the symplectic structure determine the structure of the Abelian variety though the theta functions. Due to the properties of the Abelian structure, i.e., theorem of cube, the Gauss sum is connected with another physical problem, Chern-Simons-Witten theory of the three-manifold related to some Riemann surfaces 10, 18, 39. Recently the Abelian variety (more precisely Jacobi variety), we have explicit representations 26 . Using the recent developments and our new result of the fractional Talbot phenomena, we could investigate the quantum structure over there.

On the other hand, $\theta$ function appears in my recent work on a statistical mechanical problem of closed elastic curves in a plane [24, Remark 4.3], which is closed related to the integrable system. As in the integrable system, the symplectic structure plays important roles there 13. As mentioned in 24, Introduction] in detail, the problem might be related to $\mathrm{SL}(2, \mathbb{Z})$ in replicable function theory and monstrous moonshine phenomena [19]; it might be also associated with another physical problem, the Witten 24-manifold. There the concrete relation among the symplectic structure and $\mathrm{SL}(2, \mathbb{Z})$ are also one of the central theme of the studies [19,24]. The elastic curve problem could be extended as higher dimensional objects using the Dirac operator case as in 23, references therein]. Even in the case, the theta functions are defined using the integrable system and then we should consider a connection between symplectic structure and wave properties when we consider some quantization 22. 
I believe that my interpretation of the fractional Talbot phenomena must have crucial effects on these studies.

Acknowledgements I thank K. Tamano, N. Konno and H. Mitsuhashi for the lectures related to [8] at Yokohama national university, discussions, and continuous encouragements. I am also grateful to Y. Ônishi for some discussions and encouragements and J. McKay for some discussions and telling me the reference 27.

Further I appreciate the referees for critical suggestions and references [2, 16, 31, 43.

\section{References}

1. M. V. Berry, and E. Bodenschatz, Caustics, multiply reconstructed by Talbot interference, J. Mod. Optics, 46 (1999) 349-365.

2. D. Bigourd, B. Chatel, W. P. Schleich and B. Girard, Factorization of Numbers with the temporal Talbot effect: Optical implementation by a sequence of shaped ultrashort pulses, arXiv0709.1906, .

3. M. V. Berry and S. Klein, Integer, fractional and fractal Talbot effects, J. Mod. Opt., 43 (1996) 2139-2164.

4. A. Bluher, The Weil Representation and Gauss sums, Pacific J. Math., 173 (1996) 357-373.

5. M. Born and E. Wolf, Principle of Optics, 7th edn. Pergamon, Oxford, 2001.

6. F. Cajori, A history of mathematics, Chelsea, New York, (1991).

7. G. Cliff, D. McNeilly, F. Szechtman, Weil Representations of Symplectic Groups over Rings, J. Lond. Math. Soc., 62 (2000) 423-436.

8. C. W. Curtis and I. Reiner, Representation Theory of Finite Groups and Associative Algebra, John Wiley \& Sons, New York, 1962.

9. C. W. Curtis and I. Reiner, Methods of Representation Theory vol. I, John Wiley \& Sons, New York, 1990.

10. F. Deloup, Linking forms, reciprocity for Gauss sums and invariants of 3-manifolds, Trans. Amr. Math. Soc., 351 (1999) 1895-1918.

11. C. F. Gauss, Neue Beweise und Erweiterungen des Fundamentalsatzes in der Lehre von den quadratischen Resten, 1818, 496-510, in Arithmetische Untersuchungen, , New York, Chelsea, (1965).

12. C. F. Gauss, Dioptrische Untersuchungen, Abhandlungen der Königlichen Gesellschaft der Wissenschaften in Göttingen, (1840) 1-34.

13. V. Guillemin and S. Sternberg, Symplectic techniques in physics, Cambridge Univ. Press, Cambridge, 1984.

14. J. H. Hannay and M. V. Berry, Quantization of linear maps of a torusFresnel diffraction by periodic grating, Physica, 1D 267-290 (1980).

15. E. Hecke, Lectures on the Theory of Algebraic Numbers GTM 77, Springer, Berlin, (1981).

16. S. Feng, K. Halterman and P. L. Overfelt, Subwavelength fractional Talbot effect in layered heterostructures of composite metamaterials, Phys. Rev. E, 74036612 (2006), arXiv:physics/0608003

17. K. Ireland and M. Rosen, A Classical Introduction to Modern Number Theory, 2nd ed., Springer, Berlin, (1990).

18. L. C. Jeffrey, Chern-Simons-Witten Invariant of Lens Spaces and Torus Bundles and the semiclassical approximation, Comm. Math. Phys., 147 (1992) 563-604.

19. J. McKay, Essentials of monstrous moonshine, Adv. Std. Pure. Math., 32 (2001) 347-353.

20. V. P. Kandidov and A. V. Kondrat'ev, Talbot effect in Gaussian optical systems, Quantum Elec., 31 (2001) 1032-1034.

21. G. Lion and M. Vergne, The Weil representation, Maslov index and Theta series, Birkhäuser 1980 
22. S. Matsutani, . On density of state of quantize Willmore surface: A way to a quantized extrinsic string in $\mathbb{R}^{3}$, J. Phys. A, 31 (1998) 3595-3606.

23. S. Matsutani, . Generalized Weierstrass Relations and Frobenius Reciprocity, Math. Phys. Anal. Geom., 9 (2006) 353-369.

24. S. Matsutani, . The relations in quantize elastica, J. Phys. A, 41 (2008) 075201.

25. S. Matsutani and Y.Ônishi, . Wave-particle complementarity and reciprocity of Gauss sums on Talbot effects, Found. Phys. Lett., 16 (2003) $325-341$.

26. S. Matsutani and E. Previato, . Jacobi inversion on strata of the Jacobian of the $C_{r s}$ curve $y^{r}=f(x)$, to appear J. Math. Soc. Jpn., 60 (2008).

27. Y. I. Manin, Mathematics as Metaphor, AMS, Rhode Island, (2007).

28. K. I. Oskolkov, The valleys of shadow in Schroedinger landscape, Thee Erwin Schroedinger Intl. Inst. for Math. Phys., (preprint) (2005).

29. H. Rosu and M Planat, Cyclotomic quantum clock, Proc. ICSSUR-8, Rinton Press. (2003), 366-372, quant-ph/0312073

30. H. Raszillier and W. Schempp, Fourter optıcs from the perspective of the Heisenberg group, in Lie Methods in Optics, LNP 250 ed. by J. S. Sánchez and K. B. Wolf, (Springer, Berlin, 1985).

31. H. Rosu, J. P. Treviño, H. Cabrera and J. S. Murguía, Talbot effect for dispersion in linear optical fibers and a wavelet approach, J. Mod. Phys. B, 20 1860-1875 (2006). quant-ph/0510067.

32. A. Polishchuk, Abelian varieties, theta tunctions and the Fourier transform, Cambridge, Cambridge, 2003.

33. J-P. Serre, Linear representations of Finite Group, Springer, Berlin, 1971.

34. J. Schulte, Harmonic analysis on finite Heisenberg groups, Eur. J. Comb., 25 (2004) 327-338.

35. L. S. Schulman, Techniques and Applications of Path Integration, John Wiley, New York, 1985.

36. F. Szechtman, Quadratic Gauss sums over finite commutative rings, J. Number Theory, 95 (2002) 1-13.

37. W.H.F. Talbot, Facts relating to optical sciences. no. IV, Philos. Mag., 9 401-407 (1836).

38. W.H.F. Talbot, Correspondence of William Henry Fox Talbot at Glasgow University http://foxtalbot.dmu.ac.uk/project/project.html

39. V. Turaev, Reciprocity for Gauss sums on finite abelian groups, Math. Proc. Camb. Phil. Soc, 124 (1998) 205-214.

40. V. S. Vladimirov, I. V. Volvixh, E. I. Zelenov, P-adic analysis and mathematical physics, World Scientific, Singapore, (1994).

41. A. Weil, Sur certaains groups d'operateurs unitaries, Acta Math., 11 (1964) 143-211.

42. A. Weil, Number theory for beginners, Springer-Verlag, New York, (1979).

43. J. T. Winthrop and C. R. Worthington, Theory of Fresnel images I, plane periodic objects in monochromatic light, J. Opt. Soc. Am., 55 373-381 (1965).

44. E. T. Whittaker and G. N. Watson, A course of mordern analysis, Cambridge, Cambridge, (1927). 